\title{
The effect of one-electron reduced drugs on hepatic aconitase activity and triglycerides level
}

\author{
Magdalena Wnukowska ${ }^{1}$ Slawomir Mandziuk ${ }^{2}$, Agnieszka Korga ${ }^{1^{*}}$, \\ Barbara Jodlowska-Jedrych ${ }^{3}$, Wlodzimierz Matysiak ${ }^{3}$, Justyna Halasa ${ }^{4}$, \\ Franciszek Burdan ${ }^{4,5}$, Magdalena Iwan ${ }^{1}$, Renata Gieroba ${ }^{1}$, Jaroslaw Dudka ${ }^{1,6}$ \\ ${ }^{1}$ Independent Medical Biology Unit, Medical University of Lublin, Jaczewskiego 8, 20-950 Lublin, Poland \\ ${ }^{2}$ Department of Pneumology, Oncology and Alergology, Medical University of Lublin, Jaczewskiego 8, 20-090 Lublin, Poland \\ ${ }^{3}$ Department of Histology and Embryology, Medical University of Lublin, Radziwillowska 11, 20-080 Lublin, Poland \\ ${ }^{4}$ Department of Anatomy, Medical University of Lublin, Jaczewskiego 4, 20-090 Lublin, Poland \\ ${ }^{5}$ St. John's Cancer Center, Jaczewskiego 7, 20-090 Lublin, Poland \\ ${ }^{6}$ Chair and Department of Toxicology, Medical University of Lublin, Chodzki 8, 20-093 Lublin, Poland
}

\section{ARTICLE INFO}

Received 03 December 2014 Accepted 22 December 2014

\section{Keywords:}

doxorubicin,

thyroxin,

liver,

redox equilibrium,

lipid metabolism.

\begin{abstract}
The redox cycle triggered by one electron reduction of doxorubicin and tirapazamine both anticancer agents - leads to superoxide production. This superoxide production itself removes one iron atom from the $[4 \mathrm{Fe}-4 \mathrm{~S}]$ cluster, being an active center of aconitase. In addition, the incurred changes in cell redox equilibrium may affect lipid metabolism. The aim of the study was to evaluate a concomitant effect of both drugs on hepatic aconitase activity and triglycerides level. In our study, doxorubicin (1.8 mg/kg b.w.) was administered intraperitoneal (i.p.) six times, once a week, within male Wistar rats, to achieve a cumulative dose of $10.8 \mathrm{mg} / \mathrm{kg}$ b.w. Two hours before every doxorubicin administration, tirapazamine in the dose of either 5 or $10 \mathrm{mg} / \mathrm{kg}$ b.w. was also i.p. injected. A week after withdrawing drug administration, the liver was taken for biochemical analysis. Therein, an increase in aconitase activity and a decrease in triglycerides level was seen in all groups exposed to doxorubicin. Our work demonstrated that tirapazamine administration had no influence on both tested parameters, but its higher dose rate normalized aconitase activity affected by doxorubicin.
\end{abstract}

\section{INTRODUCTION}

The main changes in normal cells caused by administration of the anticancer drug doxorubicin (DOX) is related to redox balance disorders. Therein, the mitochondria are the primary site of anthracycline-induced ROS formation [14]. Tirapazamine (TP), designed especially to kill the hypoxic tumor cells which are commonly resistant to conventional radio- and chemotherapy [4,9], engenders the same redox metabolic changes as DOX. The drug has a moderate antitumor activity $[13,16]$, but, as has been demonstrated in many experimental studies, in combination with classical chemotherapeutics and radiotherapy, it can enhance the anticancer effectiveness, when compared to treatment

\begin{tabular}{l} 
* Corresponding author \\
e-mail: a.korg@interia.pl; \\
tel.: +48 (81) 7187329 \\
\hline
\end{tabular}

with chemotherapeutics alone $[6,10,11]$.Both TP and DOX induce similar redox-related metabolic transformations. The metabolic pathway of TP in normoxic conditions, as well as that of DOX, are related to one-electron reduction with the formation of radicals, $\mathrm{TP}^{\bullet-}$ or $\mathrm{DOX}^{\bullet}$, respectively $[2,15,18]$. Indeed, many NADPH and NADH-dependent enzymes catalyzing such a reaction are common for DOX and TP [15]. Subsequent to the previous, $\mathrm{TP}^{\bullet}$ and $\mathrm{DOX}^{\bullet}$ are re-oxygenated to a non-radical parent compound, and at the same time, a superoxide radical is formed $\left(\mathrm{O}_{2}{ }^{--}\right)$. These enzymes are present especially in an abundant amount in hepatocytes [7]. A constantly repeated redox cycle may deplete NADH and NADPH quantities, hence resulting in redox balance disorders. The changes in NAD+/NADH, $\mathrm{NADP}+\mathrm{NADPH}$ ratios may then have an impact on lipid metabolism. However, NADPH is also necessary in antioxidative defense. Moreover, $\mathrm{O}_{2}{ }^{--}$removes one iron atom 
from the labile [4Fe-4S] cluster of the mitochondrial aconitase active center [8], leading to reversible inactivation of the enzyme [12]. The reversible inactivation of mitochondrial aconitase, therefore, serves as a marker of basal or anthracycline-augmented $\mathrm{O}_{2}{ }^{--}$formation in the mitochondria [14].

The aim of the present work was to evaluate the effect of tirapazamine on rat hepatic aconitse, and further, to examine possible changes in hepatic triglycerides level through its administration.

\section{MATERIALS AND METHODS}

Animals and treatment. The study was approved by the Local Ethical Committee at the Medical University of Lublin. The subject male Wistar rats (150-180 g) were kept under conventional laboratory conditions (temperature $22^{\circ} \mathrm{C}$, humidity $60-70 \%, 12 \mathrm{~h} \mathrm{light/dark} \mathrm{cycle)} \mathrm{and} \mathrm{fed}$ with standard rodent granulated fodder LSM ${ }^{\circledR}$ (AGROPOL, Poland). Food and water were freely available. The animals were randomly placed into either one of five experimental groups or one control group. The experimental rats received intraperitoneal tirapazamine (Advanced Tech. \& Ind. Co., Ltd; China) in either doses 5 or $10 \mathrm{mg} / \mathrm{kg}$ - two hours before doxorubicin administrations. Doxorubicin (EBEWE Arzneimittel Ges.m.b.H., Austria) was given once a week in a dose of $1.8 \mathrm{mg} / \mathrm{kg}$ for 6 weeks. One week after the last dose of doxorubicin (cumulative dose $10.8 \mathrm{mg} / \mathrm{kg} \mathrm{b.w.),}$ liver samples were taken during pentobarbital anesthesia and the animals were sacrificed by decapitation. The excised liver samples was kept at $-70^{\circ} \mathrm{C}$ until biochemical analysis. The hepatic concentration of triglycerides (Cormay, Polska) and aconitase activity (Cayman, USA) were determined according to standard procedures, using plate reader PowerWaveXS (Bio-Tek, USA).

Statistical analysis. The obtained data were analyzed using STATISTICA 5.0. (Statsoft Inc., USA). Statistical significance was evaluated by U Mann-Witney test (vs. saline control) and by one-way analysis of variance (KruskalWallis ANOVA). The post-hoc test (Newman-Keuls) was used to verify the null hypothesis, according to which the tirapazamine administration influenced the evaluated parameters in rats receiving doxorubicin. All data are expressed as mean \pm standard deviation. The value of $p \leq 0.05$ was considered statistically significant.

\section{RESULTS}

One week after drug cessation, the activity of aconitase was significantly higher in the livers of the rats which received doxorubicin. The activity of the enzyme was also elevated in rats administered tirapazamine, but such changes were insignificant. Interestingly, tirapazamine significantly normalized aconitase activity in rats treated with doxorubicin $(\mathrm{p} \leq 0.05$ among DOX and DOX $+10 \mathrm{TP})$. Furthermore, the activity of the enzyme in both groups of DOX+TP was constantly higher, in comparison to the control.
Table 1. Hepatic aconitase activity $(\mu \mathrm{mol} / \mathrm{min} / \mathrm{ml})$ in rats receiving doxorubicin (DOX) and tirapazamine (TP)

\begin{tabular}{|c|c|c|c|c|c|c|c|}
\hline & $\mathrm{N}$ & $\mathrm{M}$ & $\mathrm{Me}$ & Min & Max & SD & $\mathrm{p}$ \\
\hline Control & 8 & 0.468 & 0.365 & 0.173 & 1.226 & 0.339 & \\
\hline DOX & 5 & 1.173 & 1.195 & 0.817 & 1.446 & 0.254 & $0.013^{*}$ \\
\hline 5TP & 5 & 0.596 & 0.589 & 0.424 & 0.723 & 0.124 & 0.107 \\
\hline $10 T P Z$ & 6 & 0.927 & 1.045 & 0.306 & 1.320 & 0.417 & 0.052 \\
\hline DOX+5TP & 5 & 0.951 & 1.092 & 0.582 & 1.108 & 0.231 & $0.040^{*}$ \\
\hline DOX+10TP & 6 & $0.776 \neq$ & 0.747 & 0.487 & 1.195 & 0.282 & $0.039 *$ \\
\hline
\end{tabular}

$* \mathrm{p} \leq 0.05$ vs Control; $\$ \mathrm{p} \leq 0.05$ vs DOX

Our experimental results revealed that the level of triglycerides in the liver was significantly reduced by doxorubicin. Moreover, tirapazamine had an insignificant effect on these parameters. What is more, tirapazamine had no effect on hepatic triglycerides in rats receiving doxorubicin.

Table 2. Hepatic triglycerides level $(\mathrm{mg} / \mathrm{dl}$ of homogenate supernatant) in rats receiving doxorubicin (DOX) and tirapazamine (TP)

\begin{tabular}{|c|c|c|c|c|c|c|c|}
\hline & N & M & Me & Min & Max & SD & $p$ \\
\hline Control & 8 & 193.69 & 172.59 & 149.83 & 268.37 & 40.89 & \\
\hline DOX & 6 & 98.78 & 101.94 & 73.97 & 128.02 & 19.48 & $0.001^{*}$ \\
\hline 5TPZ & 6 & 198.67 & 207.68 & 111.90 & 260.78 & 54.58 & 0.848 \\
\hline $10 T P Z$ & 6 & 146.67 & 145.09 & 90.09 & 209.57 & 40.38 & 0.053 \\
\hline DOX+5TP & 6 & 96.41 & 82.03 & 54.05 & 172.59 & 43.58 & $0.001^{*}$ \\
\hline DOX+10TP & 6 & 109.69 & 98.15 & 76.81 & 190.61 & 41.09 & $0.002 *$ \\
\hline
\end{tabular}

* $\mathrm{p} \leq 0.05$ vs Control

\section{DISCUSSION}

The main result of the study is that the work demonstrated an increase of hepatic aconitase activity and a decrease of triglycerides level in all experimental groups after one week from last dose of doxorubicin. Of note, tirapazamine administration alone in both tested doses had an insignificant effect on both tested parameters, but the higher dose of this agent normalized aconitase activity affected by doxorubicin.

Supplementation of doxorubicin anticancer therapy by tirapazamine seems rational, since there are normoxic and hypoxic phenotype population cells resulting from differences in oxygen concentration in different part of a tumor. Such procedure might be more efficient than doxorubicin monotherapy $[6,10,11]$.

Doxorubicin and tirapazamine are involved in a enzymatic NADH and NADPH-related one electron redox cycle that leads to free radical generation $[2,18]$. Because the enzymes produce free radicals in the presence of doxorubicin at a several times higher activity in the liver than in other organs [7], in the current study, the model with cumulative dose was used, and biochemical evaluation was performed one week after the last doxorubicin injection. We put forward that simultaneous disturbance in NADPH and NADH levels and free radical production may cause changes in redox-dependent metabolism. It is well known that lipids oxidation is strictly related to the NADH/NAD+ ratio, while lipids (cholesterol) synthesis is dependent upon 
the NADPH/NADP+ ratio. Hence, the ratio of NADPH/ $\mathrm{NADP}+$ also plays a pivotal role in the defense against free radical overproduction. This overproduction of $\mathrm{O}_{2}^{-}$observed during one electron redox cycle of both drugs, may regulate the activity of aconitase - a key enzyme within the Krebs cycle - via removing one iron atom from the labile [4Fe-4S] cluster of the aconitase center [8], leading to the reversible inactivation of the enzyme [12]. The reversible inactivation of mitochondrial aconitase, therefore, serves as a marker of basal or anthracycline-augmented $\mathrm{O}_{2}^{--}$formation in the mitochondria [14].

As it was prior mentioned, in our experiment, the aconitase activity was not reduced, but elevated, in all groups where doxorubicin was administered (DOX, DOX $+5 \mathrm{TP}$ and DOX+10TP). The activity of this enzyme was also elevated in rats receiving tirapazamine, but these changes were insignificant. The reverse results to our expectations may be an adaptive reaction. Thus, when medicament generating superoxide are present in the cell, an inhibition of aconitase is observable [14]. Because the doxorubicin halflife (T1/2) is $30 \mathrm{~h}$ [17], the drug is practically excreted from the organism after one week. However, during the period of six weeks when doxorubicin was administered every week, an adaptive increase in hepatic aconitase protein and related secondary activity might have appeared. Hence, the cumulative effect of both tested drugs was not revealed. Aconitase and triglycerides are linked because the acetyl CoA bettaoxidation product is subsequently transformed by aconitase. Our experiment revealed that the level of triglycerides in the liver was significantly reduced by doxorubicin, however, tirapazamine administration had an insignificant effect on this parameter. Moreover, tirapazamine administration had no effect on hepatic triglycerides in the rats receiving doxorubicin. A similar effect for doxorubicin was observed in our previous studies [5], where rats received this drug once a week in a higher cumulative dose $(18 \mathrm{mg} / \mathrm{kg})$, and where the liver was taken three weeks after completing drug administration. Of note, the results of this work is interesting in light of another mechanism - in that doxorubicin inhibits the transport of fatty acid to the mitochondria [1]. Consequently, the augmentation of cytoplasmic fatty acid levels usually leads to an increase in triglycerides production, thus the hepatic level of triglycerides should increase. Yet, in contrary to speculation, the results reveal a reverse effect a decrease in hepatic triglycerides level. These results are consistent with results published by Bizzi et al. [3], who reveal a reduced triglycerides level in the liver of rats even 14-21 days after administering a single toxic dose of doxorubicin $(7.5 \mathrm{mg} / \mathrm{kg}$ i.v.). Simultaneously, the authors indicated massive plasma hyperlipemia. Hence, prospective studies might clarify the mechanism of lipid disturbance in the blood and liver affected by doxorubicin. In conclusion, our work showed that tirapazamine administration did not influence both tested parameters, but its higher dose normalized aconitase activity affected by doxorubicin. However, other studies - including those not based upon rodent life-forms - are needed before such polidrug therapy is introduced to daily human clinical practice.

\section{REFERENCES}

1. Abdel-aleem S. et al.: Acute and chronic effects of adriamycin on fatty acid oxidation in isolated cardiac myocytes. J. Mol. Cell Cardiol., 29, 789, 1997.

2. Bachur N.R., Gordon S.L., Gee M.V.: A general mechanism for microsomal activation of quinone anticancer agents to free radicals. Cancer Res., 38, 1745, 1978.

3. Bizzi A. et al.: Adriamycin causes hyperlipemia as a consequence of nephrotoxicity. Toxicol. Lett., 18, 291, 1983.

4. Brown J.M.: The hypoxic cell: a target for selective cancer therapyeighteenth Bruce F. Cain Memorial Award lecture. Cancer Res., 59, $5863,1999$.

5. Czuba B. et al.: The effect of thyroxin on hepatic redox equilibrium and lipid metabolism in rats treated with doxorubicin. Curr Issues Pharm Med Sci., 27(4), 220, 2014.

6. Dorie M.J., Brown J.M.: Modification of the antitumor activity of chemotherapeutic drugs by the hypoxic cytotoxic agent tirapazamine. Cancer Chemotherapy and Pharmacology., 39, 361, 1997.

7. Dudka J. et al.: Activity of NADPH-cytochrome P-450 reductase of the human heart, liver and lungs in the presence of (-)-epigallocatechin gallate, quercetin and resveratrol: an in vitro study. Basic Clin. Pharmacol. Toxicol., 97, 74, 2005.

8. Hausladen A., Fridovich I.: Superoxide and peroxynitrite inactivate aconitases, but nitric oxide does not. J. Biol. Chem., 269, 29405, 1994.

9. Hong B. et al.: Hypoxia-targeting by tirapazamine (TPZ) induces preferential growth inhibition of nasopharyngeal carcinoma cells with Chk1/2 activation. Investigational New Drugs., 29, 401, 2011.

10. Jounaidi Y. and Waxman D.J.: Combination of the bioreductive drug tirapazamine with the chemotherapeutic pro-drug cyclophosphamide for P450/P450-reductase-based cancer gene therapy. Cancer Res., 60, 3761, 2000.

11. Marcu L., Olver I.: Tirapazamine: from bench to clinical trials. Current Clin. Pharmacol., 1, 71, 2006.

12. Minotti G. et al.: Anticancer anthracyclines and the control of iron metabolism: quinine-dependent and independent mechanisms. Methods Enzymol., 378, 340, 2004.

13. Reddy S.B., Williamson S.K.: Tirapazamine: a novel agent targeting hypoxic tumor cells. Expert Opinion on Investigational Drugs., 18, 77, 2009.

14. Salvatorelli E. et al.: Defective one or two electron reduction of the anticancer anthracycline epirubicin in human heart. Relative importance of vesicular sequestration and impaired efficiency of electron addition. J. Biol. Chem., 281, 10990, 2006.

15. Salvatorelli E. et al.: Pharmacokinetic Characterization of Amrubicin Cardiac Safety in an Ex Vivo Human Myocardial Strip Model. II. Amrubicin Shows Metabolic Advantages over Doxorubicin and Epirubicin. JPET., 341, 2474, 2012.

16. von Pawel J. et al.: Tirapazamine plus cisplatin versus cisplatin in advanced non-small-cell lung cancer: a report of the international CATAPULT I study group. Cisplatin and tirapazamine in subjects with advanced previously untreated non-small-cell lung tumors. J. Clin. Oncol., 18, 1351, 2000.

17. Warren K.E. et al.: Effect of fluconazole on the pharmacokinetics of doxorubicin in nonhuman primates. Antimicrob. Agents Chemother., 44, 1100, 2000.

18. Wouters B.G. et al.: Mitochondrial dysfunction after aerobic exposure to the hypoxic cytotoxin tirapazamine. Cancer Res., 61, 145, 2001. 